\title{
The Effects of a Multifaceted Intervention to Improve Venous Thromboembolism Prophylaxis are Sustained Over Time
}

\author{
Robert C. Pendleton ${ }^{1}$, Matthew T. Rondina ${ }^{1, *}$, Michelle Wheeler ${ }^{2}$ and George M. Rodgers ${ }^{1,3}$ \\ Departments of Internal ${ }^{1}$ Medicine, ${ }^{2}$ Pharmacy and ${ }^{3}$ Pathology, The University of Utah Health Sciences Center, Salt \\ Lake City, Utah, UT 84132, USA
}

\begin{abstract}
Background: The majority of venous thromboembolic events occur in hospitalized patients. Although there are several effective regimens, venous thromboembolism (VTE) prophylaxis is still under-utilized in medically-ill patients. In 2003 a multi-faceted quality improvement intervention was implemented to improve the use of VTE prophylaxis. We previously described the significant improvements in VTE prophylaxis in the two years following this intervention.

Objective: To determine the longitudinal effects of this quality improvement intervention in prescribing practices for VTE prophylaxis in medically-ill patients.

Methods: Since the original intervention in 2002, the only on-going maintenance has been 4 annual lectures. We collected data on 121 medically-ill patients hospitalized in 2006, including risk factors for VTE, pharmacologic agent and dose prescribed (or mechanical devices, if uses), and timing of prophylaxis in relation to admission.

Results: Overall, $83 \%$ were at high-risk for VTE and 77\% received prophylaxis, a significant improvement compared to the 2002 pre-intervention cohort $(40 \%, \mathrm{p}<0.001)$ and similar to the 2003 post-intervention cohort $(72 \%)$. Significantly more patients received preferred regimens for VTE prophylaxis in 2006 compared to 2002. Pneumatic compression devices, unfractionated heparin (UFH) 5,000 units TID, low molecular weight heparin (LMWH), and UFH 5,000 units BID were prescribed in $28 \%, 40 \%, 38 \%$, and $3 \%$ respectively. Prophylaxis was prescribed within the first 24 hours of admission in $88 \%, 24-48$ hours in $8 \%$, and after 48 hours in $4 \%$.

Conclusions: We have demonstrated that a multifaceted quality improvement intervention leads to a sustained improvement in the use of preferred VTE prophylaxis prescribing practices. The maintenance of these effects has been minimal, consisting of four 1-hour long educational sessions per year targeted to clinical pharmacists and medicine residents.
\end{abstract}

Key Words: Venous thromboembolism, prophylaxis, prevention, quality Improvement, education.

\section{INTRODUCTION}

Every year in the United States venous thromboembolism (VTE), including deep vein thrombosis (DVT) and pulmonary embolism (PE), is recognized in over 250,000 people and accounts for an estimated 100,000 deaths [1]. The majority of these events occur with hospitalization or surgery [2]. Routine preventive measures are effective in reducing VTE and evidence-based guidelines are available to guide prophylaxis decisions in these patients [2]. Yet, VTE prophylaxis is still under-utilized. For example, only $29 \%$ of patients in the DVT Free Registry had received some form of prophylaxis in the 30 days prior to their event [3]. Other studies have demonstrated low prophylaxis rates in at-risk hospitalized patients $[4,5]$. This failure to routinely deliver effective prophylaxis in at-risk patients has led to increasing attention by regulatory authorities, including the Agency for Healthcare Research and Quality (AHRQ)[5] and National Quality Forum (NQF) [6].

Many studies have formally evaluated methods to improve VTE prevention [7], including computer decision

*Address correspondence to this author at the Department of Internal Medicine, University of Utah, 50 North Medical Drive, Room 4B120, SLC, UT 84132, USA; Tel: (801) 581-7818; Fax: (801) 585-9166;

E-mail: matthew.rondina@hsc.utah.edu support, written protocols, audit-feedback, educational sessions, and passive approaches (i.e. availability of guidelines). Although computer decision support or electronic alerts improve VTE prevention and lead to a reduction in VTE events [8], many institutions do not have computer decision support capabilities. For these and other institutions, multifaceted interventions have been advocated as a preferred approach [9], but have not been adequately studied.

We previously reported the effectiveness of a multifaceted quality improvement (QI) intervention (institutional audit feedback, written protocols, and education) to improve short-term VTE prevention practices in medically-ill patients [10]. However, the longitudinal effects of this type of intervention are unknown. Knowledge of the lasting effects of a QI intervention could have important implications to individual institutions so that adequate resources can be allocated. In this study, we report a 4-year follow up analysis of VTE prevention practices after our multifaceted QI intervention.

\section{MATERIALS AND METHODOLOGY}

\section{Study Design}

This is a single-center prospective cohort study. Retrospective data collection was chosen to minimize potential 
influence on prescribing practices. IRB consent was obtained and a waiver of informed consent was granted by the Institutional Review Board (IRB). Original analysis and methods have been published previously[10]. Briefly, the University of Utah Health Sciences Center is a tertiary care center with multi-disciplinary teams caring for patients, including resident and supervising physicians, nurses, and pharmacists. In 2002 we performed a retrospective analysis of VTE prevention practices in patients admitted to the pulmonary, cardiology, oncology, and general medical services. The oncology, cardiology, and pulmonary services are attended by specialists while academic hospitalists attend the general medicine service. After demonstrating poor VTE prevention rates in the 2002 analysis [10], we implemented a multifaceted intervention (outlined below). Data collected in 2003 demonstrated significant improvement in VTE prevention rates [10]. The current analysis is a 4-year follow-up of the original intervention and excluded oncology service patients as this service was moved to a stand-alone cancer center.

\section{Patient Selection}

Data was collected on consecutive patients $\geq 18$ years with a hospital length of stay of $>48$ hours admitted to the cardiology, pulmonary, or general medical services. Admission dates were a sample of two different two-week blocks (January 1-14, 2006 and April 1-14, 2006). These blocks were chosen to be consistent with dates of analysis in the pre-intervention cohort. Patients receiving therapeutic anticoagulation were excluded. The current year was chosen for analysis as all residents involved in the original intervention (2002) had matriculated and thus the residents currently training were not involved in the original intervention.

\section{Intervention Design}

In 2002 a pre-intervention data analysis was followed by a multifaceted QI intervention: (1) a risk-assessment tool completed at the time of a hospital admission, (2) structured educational sessions on appropriate VTE prevention (including both pharmacologic agents and mechanical devices), and (3) audit-feedback where institutional data was reviewed during the educational sessions. The risk-assessment tools, education sessions, and study protocols were designed by a team of thrombosis specialists, including two physicians (R.P. \& G.R.) and a pharmacist (M.W.), in accordance with guideline recommendations [11]. The educational sessions were given once to the clinical pharmacists by an anticoagulation pharmacist (M.W.) and on three separate occasions to the medicine house officers by a physician (R.P.).

In 2003, after post-intervention data analysis, the written protocol was disbanded due to lack of routine use. Educational sessions were continued and consisted of institutional prophylaxis rates (audit feedback) along with an evidencebased review of VTE prophylaxis. In 2004 to 2006, evidence-based educational sessions were continued, but audit feedback data was no longer included in the presentations. Annually from 2003-2006, these educational sessions were given once to the clinical pharmacists by an anticoagulation pharmacist (M.W.) and on three separate occasions to the medicine house officers by a physician (R.P.).

\section{Statistical Analysis}

Statistical comparisons between years 2002 and 2006 were made using the chi-square test or Fisher's exact test, as appropriate. All p-values are for a two-sided comparison. Significance was set at a $\mathrm{p}<0.05$.

\section{RESULTS}

One hundred and twenty one patients met inclusion criteria. Patient demographics are listed in Table 1. Eighty-three percent of patients were considered high-risk for VTE, 71\% had $\geq 1$ major and $12 \%$ had $\geq 2$ minor risk factors for VTE, and $6 \%$ of patients had no recognized risk factors. Prophylaxis was prescribed in $77 \%$ of these high-risk patients. This improvement was sustained and significant from 2002 and similar to the 2003 post-intervention cohort (Fig. 1, p<0.001). Additionally, $60 \%$ of low risk patients received prophylaxis. Timing of prophylaxis in relation to admission was available for 90 patients. Prophylaxis was prescribed within the first 24 hours of admission in $88 \%, 24-48$ hours in $8 \%$, and after 48 hours in $4 \%$. Pneumatic compression devices were used in $28 \%$ of patients. Pharmacologic agents were used more commonly. Unfractionated heparin (UFH) 5,000 units TID, low molecular weight heparin (LMWH), and UFH 5,000 units BID were prescribed in $40 \%, 38 \%$, and $3 \%$ respectively. There were significantly more patients receiving preferred pharmacologic regimens (UFH 5,000 units TID or LMWH 40mg daily) in 2006 compared to the 2003 postintervention and 2002 pre-intervention cohort (78\%, 45\%, and $10 \%$ respectively, $\mathrm{p}<0.001)$. Patients on the general medicine service were significantly more likely to receive prophylaxis compared to patients on the specialty services (93\% versus $47 \%, \mathrm{p}<0.001)$.

\section{DISCUSSION}

Despite the availability of effective preventive measures, VTE prevention is often underutilized. Our results demonstrate that a multifaceted quality improvement intervention designed by pharmacists and physicians and consisting of written protocols, education, and audit-feedback produces sustained effects over time. Of interest, these improvements in VTE prevention were sustained despite phasing out the written protocol and audit-feedback components. Sustained longitudinal effects were noted in both the use of prophylaxis in at-risk patients as well as the type of prophylaxis utilized.

Prophylaxis rates were high for both high-risk and lowrisk patients, although prophylaxis was more likely to be prescribed for the high-risk group. Importantly, with our intervention, prophylaxis use increased in both high-risk and low-risk groups. It is possible that the use of prophylaxis in this latter group was due to risk factors that were not captured by our analysis. However, a more likely explanation is that for many prescribers, prophylaxis use became standard practice without thought of risk factor assessment. This would be problematic if VTE risk were uncommon. However, $94 \%$ of all medical patients at our institution have at least one risk factor for VTE and $83 \%$ met our pre-defined criteria for "high-risk". Because of the commonness of these risk factors, the use of VTE prophylaxis as a routine admission practice is a reasonable approach. 
Table 1. Study Population Demographics and VTE Risk Group Designation

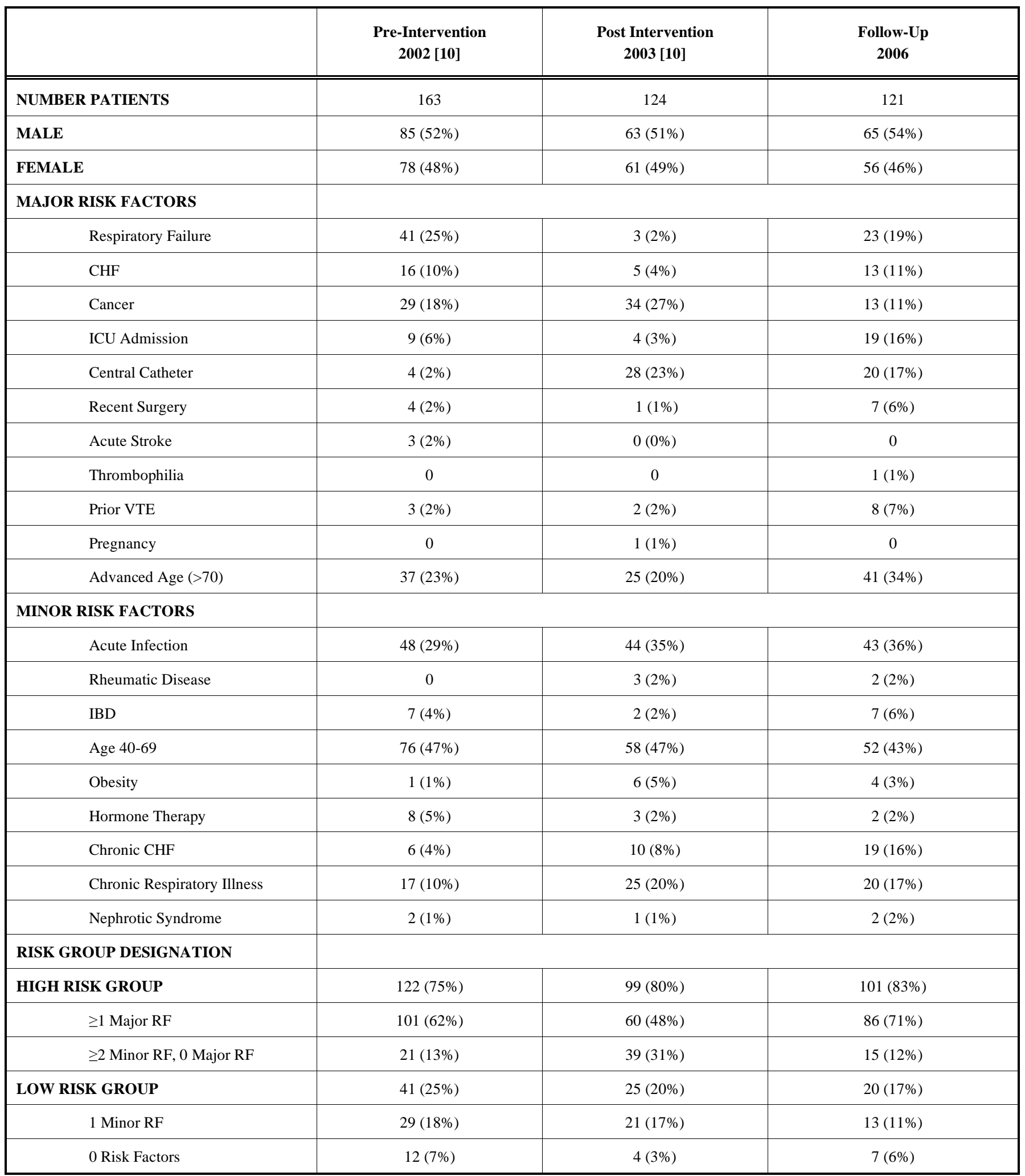

Abbreviations: CHF, congestive heart failure; ICU, intensive care unit; VTE, venous thromboembolism; IBD, inflammatory bowel disease; RF, risk factor.

A limitation of our study is that we were not able to determine, with precision, why there is a sustained longitudinal effect. In the original 2002 multifaceted intervention it was uncertain which component led to improved prophylaxis use.
The written protocol was used in a minority $(<30 \%)$ of patients and thus was not felt to be efficacious. Educational sessions were also unlikely to be the primary reason for improvement in prophylaxis as educational lectures on VTE 


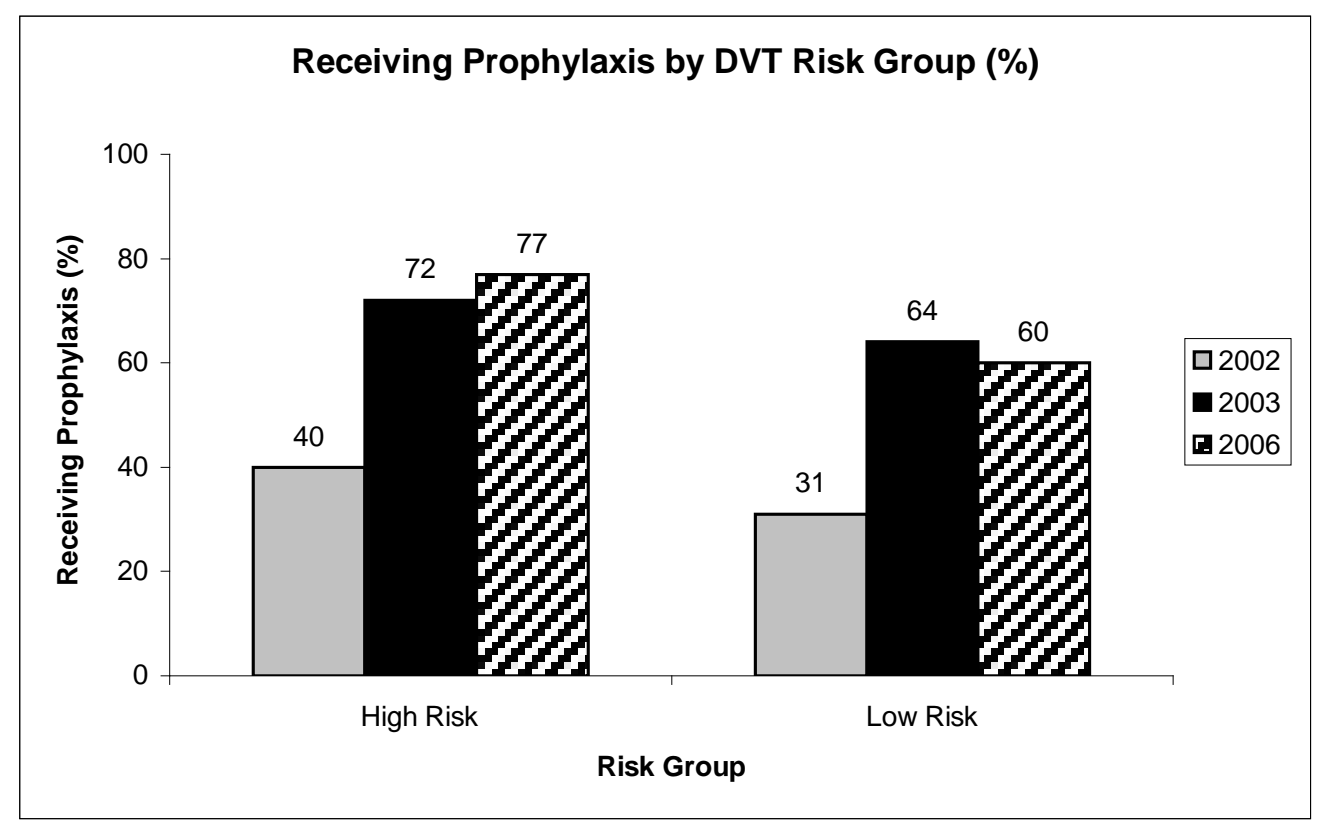

Fig. (1). VTE prophylaxis rates according to risk group (high versus low) and year. 2002 is pre-intervention, 2003 is post-intervention, and 2006 is longitudinal follow-up.

prevention had been given to the medicine residents for two years before our original pre-intervention analysis. The use of educational lectures by our anticoagulation pharmacist to clinical pharmacists may also have resulted in more pharmacist-driven recommendations for appropriate VTE prophylaxis. The audit-feedback component, in the context of educational sessions in the original intervention, may have been the most important component. If so, once a change in practice was made, ongoing audit-feedback was not necessary to produce a sustainable effect. Alternatively, the improvement in VTE prevention may reflect increased awareness nationally and not be solely due to our intervention. However, the strong temporal relationship and sustained magnitude of effect imply that the original intervention was important.

It is uncertain if the improvement in VTE prevention was due to a cultural change at the resident level or due to the influence of a stable group of academic hospitalists. In fact, prophylaxis was more likely to be prescribed by residents who were attended by a general medicine hospitalist compared to a subspecialty attending. However, $88 \%$ of the time prophylaxis was prescribed at the time of hospital admission, before the attending would have exerted their influence on the resident's management. This suggests that the routine use of prophylaxis was propagated through a cultural change in practice at the resident level.

\section{CONCLUSION}

For VTE prevention at an academic medical center, we have demonstrated that a multifaceted quality improvement intervention given by pharmacists and physicians leads to a sustained improvement in practice. The maintenance of these effects has been minimal, consisting of four 1-hour long educational sessions per year targeted to clinical pharmacists and medicine residents.

\section{TRIAL REGISTRATION}

This study has been registered and is assigned a Clinical Trials ID of NCT00810784.

\section{CONFLICT OF INTEREST}

Dr. Pendleton is on the speaker's bureau for GlaxoSmithKline and Sanofi-Aventis. Dr. Rodgers is on the speaker's bureau for GlaxoSmithKline. Dr. Wheeler has no conflicts to disclose. Dr. Rondina is on the speaker's bureau for Pfizer, GlaxoSmithKline, and Sanofi-Aventis.

\section{REFERENCES}

[1] Anderson FA, Jr, Wheeler HB, Goldberg RJ, et al. A populationbased perspective of the hospital incidence and case-fatality rates of deep vein thrombosis and pulmonary embolism. The Worcester DVT Study. Arch Intern Med 1991; 151: 933-8.

[2] Geerts WH, Pineo GF, Heit JA, et al. Prevention of venous thromboembolism: the Seventh ACCP Conference on Antithrombotic and Thrombolytic Therapy. Chest 2004; 126: 338S-400S.

[3] Goldhaber SZ, Tapson VF. A prospective registry of 5,451 patients with ultrasound-confirmed deep vein thrombosis. Am J Cardiol 2004; 93: 259-62.

[4] Anderson FA, Jr, Wheeler HB, Goldberg RJ, et al. Changing clinical practice. Prospective study of the impact of continuing medical education and quality assurance programs on use of prophylaxis for venous thromboembolism. Arch Intern Med 1994; 154: 669-77.

[5] Leape LL, Berwick DM, Bates DW. What practices will most improve safety? Evidence-based medicine meets patient safety. JAMA 2002; 288: 501-7.

[6] Forum NQ. Safe practices for better healthcare: a consensus report 2003.

[7] (ASERNIP-S) ASaERoNIP-S. Interventions to increase the uptake of venous thromboembolism prophylaxis in hospitals. Melbourne: National Institute of Clinical Studies 2003.

[8] Kucher N, Koo S, Quiroz R, et al. Electronic alerts to prevent venous thromboembolism among hospitalized patients. N Engl J Med 2005; 352: 969-77.

[9] Shojania KG, Grimshaw JM. Evidence-based quality improvement: the state of the science. Health Aff (Millwood) 2005; 24: 138-50. 
[10] Stinnett JM, Pendleton R, Skordos L, Wheeler M, Rodgers GM. Venous thromboembolism prophylaxis in medically ill patients and the development of strategies to improve prophylaxis rates. Am J Hematol 2005; 78: 167-72.
[11] Proceedings of the Seventh ACCP Conference on Antithrombotic and Thrombolytic Therapy: evidence-based guidelines. Chest 2004; vol. 126: pp. 172S-696S

(C) Pendleton et al.; Licensee Bentham Open.

This is an open access article licensed under the terms of the Creative Commons Attribution Non-Commercial License (http: //creativecommons.org/licenses/ by-nc/3.0/) which permits unrestricted, non-commercial use, distribution and reproduction in any medium, provided the work is properly cited. 